\title{
Embryonic rat vascular smooth muscle cells revisited - a model for neonatal, neointimal SMC or differentiated vascular stem cells?
}

\author{
Vascular Cell 6:6 I DOI: 10.1186/2045-824X-6-6 I c Li et al.; licensee Publiverse Online S.R.L. 2014 \\ Received: 5 Dec 2013 | Accepted: 28 Dec 2014 | Published: 15 Dec 2014 \\ Kennedy Eimear, Hakimjavadi Roya, Greene Chris, Mooney Ciaran J, Fitzpatrick Emma, Collins Laura E, \\ Loscher Christine E, Guha Shaunta, Morrow David, Redmond Eileen M, Cahill Paul A ${ }^{\circledR}$ \\ ${ }^{+}$Contributed equally ${ }^{\circledR}$ Corresponding author
}

\begin{abstract}
Background

The A10 and A7r5 cell lines derived from the thoracic aorta of embryonic rat are widely used as models of nondifferentiated, neonatal and neointimal vascular smooth muscle cells in culture. The recent discovery of resident multipotent vascular stem cells within the vessel wall has necessitated the identity and origin of these vascular cells be revisited. In this context, we examined A10 and A7r5 cell lines to establish the similarities and differences between these cell lines and multipotent vascular stem cells isolated from adult rat aortas by determining their differentiation state, stem cell marker expression and their multipotency potential in vitro.
\end{abstract}

\section{Methods}

Vascular smooth muscle cell differentiation markers (alpha-actin, myosin heavy chain, calponin) and stem cell marker expression (Sox10, Sox17 and S100ß) were assessed using immunocytochemistry, confocal microscopy, FACS analysis and real-time quantitative PCR.

\section{Results}

Both A10 and A7r5 expressed vascular smooth muscle differentiation, markers, smooth muscle alpha- actin, smooth muscle myosin heavy chain and calponin. In parallel analysis, multipotent vascular stem cells isolated from rat aortic explants were immunocytochemically myosin heavy chain negative but positive for the neural stem cell markers Sox $10^{+}$, a neural crest marker, Sox $17^{+}$the endoderm marker, and the glia marker, S100 $\beta^{+}$. This multipotent vascular stem cell marker profile was detected in both embryonic vascular cell lines in addition to the adventitial progenitor stem cell marker, stem cell antigen-1, Sca $1^{+}$. Serum deprivation resulted in a significant increase in stem cell and smooth muscle cell differentiation marker expression, when compared to serum treated cells. Both cell types exhibited weak multipotency following adipocyte inductive stimulation. Moreover, Notch signaling blockade following $\gamma$-secretase inhibition with DAPT enhanced the expression of both vascular smooth muscle and stem cell markers.

\section{Conclusions}

We conclude that A10 and A7r5 cells share similar neural stem cell markers to both multipotent vascular stem cells and adventitial progenitors that are indicative of neointimal stem-derived smooth muscle cells. This may have important implications for their use in examining vascular contractile and proliferative phenotypes in vitro.

\section{Introduction}

The arterial blood vessel is comprised of three distinct layers; an innermost monolayer of endothelial cells, a medial layer composed primarily of vascular smooth muscle cells [vSMCs] [ 1-3] and some multipotent vascular stem cells [MVSCs] [ $1,4,5]$, and an outer adventitial layer of fibroblast cells and some vSMC-related stem cell antigen-1 (Sca1 ${ }^{+}$) positive progenitor cells $[6,7]$. The medial vSMCs in the vessel wall are not terminally differentiated but can undergo "phenotypic switching" following vascular injury [ 8,9$]$. In a similar manner, vSMCs in culture are thought to be "phenotypically modulated' [ 10, 11,3]. These 'contractile' cells initially express markers for SMC differentiation such as smooth muscle $\alpha$-actin (SMA), smooth muscle myosin heavy chain (SM-MHC), calponin (CNN1) and SM-22 $\alpha$. However, proliferating vSMC display the characteristics of de-differentiated vSMCs in that they have minimal expression of SMC differentiation marker proteins and do not contract when cultured on a silicone substrate [12]. Moreover, proliferating 'synthetic' vSMC 
can be converted back or 'modulated' to a more mature phenotype by placing the cells in quiescent media to selectively increase in the expression of the SMC differentiation markers [12,13].

Original studies in canine carotid arteries suggested that 'neointimal modulated proliferative SMC' were not derived from differentiated SMC but instead formed from a myosin negative type II medial SMC cell [ 14]. Recent lineage tracing studies in vivo using SM-MHC as a marker suggest that SM-MHC ${ }^{-}$negative resident multipotent vascular stem cells [MVSCs], and not de-differentiated vSMCs, repopulate the neointima following vascular injury and proliferate and differentiate into vSMCs [ 5, 15]. Moreover, Notch activation following co-culture of MVSCs with OP9-Delta1 feeder cells for 2 weeks promoted MVSC transition to vSMC [ 5]. MVSCs are resident stem cells located in the tunica media and adventitial layers of the arterial wall and express the neural crest cell marker Sox10, endoderm marker Sox 17, glial cell marker S100 $\beta$ and neural filament-medium polypeptide (NFM) [ 5]. Sox 10 is routinely used to identify and trace MVSCs in blood vessels $[5,15]$. MVSCs can be cloned from single cells, possess telomerase activity and can differentiate into Schwann cells, peripheral neurons, vSMCs, chondrocytes, adipocytes and osteoblasts [ 5].

The A10 and A7r5 cell lines were originally derived from the thoracic aorta of 14-17 day old embryonic BD1X rats and are a commonly used model of vSMC in culture [16]. Initial characterisation of these cells suggested that they were nondifferentiated VSMC that differ from neonatal but bear significant resemblance to neointimal cells [ 16]. The functionality of A10 and A7r5 cells and their relevance to mechanisms underlying the contractile properties of highly differentiated vascular smooth muscle cells is questionable. Nevertheless, these cell lines exhibit an adult smooth muscle phenotype and show expression and promoter activity of several highly restricted smooth muscle cell markers [ 17]. Moreover, a phenotypic transition from vascular smooth to skeletal muscle and a detailed examination of the gene expression program associated with this transition has been reported [18]. The cells also have the ability to contract by both calciumdependent and -independent mechanisms [ 19]. On the other hand, the actin cytomatrix of these cells shows many structural similarities to fibroblasts, much like other smooth muscle cell types that revert to a less differentiated phenotype in culture [ $1,16,17]$. Despite this, the cell lines are widely used by researchers due to their apparent similarities to neointimal cells and therefore offer an excellent model system for studying the transcriptional regulation of vSMC markers and signaling cascades involved in neointima formation $[16,17]$.

In light of the recent characterization of resident vascular stem cells within vascular medial and adventitial regions and their transition to vSMC following vascular injury [ 5, 20], it has been suggested that traditionally defined proliferative/synthetic vSMCs, such as A10 and A7r5 cell lines may be derived from the differentiation of resident stem cells in culture rather than the de-differentiation of immature/mature vSMCs [ 15,5]. As both A10 and A7r5 are derived from embryonic tissue, both cell lines were examined for their stem marker expression with a view to investigating whether these vSMC cell lines share characteristics with resident vascular stem cells in culture.

\section{Materials and methods}

\section{Materials}

All materials were of the highest purity commercially available. Primary antibodies included: SMA (monoclonal mouse anti- $\alpha$-actin antibody, Sigma Cat No: A5228), SM-MHC (monoclonal mouse anti-myosin antibody, Sigma Cat No: clone hSM-V, M7786), (anti-MHC antibody [1G12], Abcam Cat No: Ab683) and (the goat polyclonal MYH11 Antibody (N-16) from Santa Cruz, Cat No: SC79079 ), CNN1 (monoclonal mouse anti-calponin antibody, Sigma Cat No: C2687), Sox10 (monoclonal rabbit anti-Sox10 antibody, Abcam Cat No: ab155279), Sox17 (monoclonal rabbit anti-Sox17 antibody, Millipore Cat No: 09-038) and S100 $\beta$ (monoclonal rabbit anti-S100 $\beta$ antibody, Millipore Cat No: 04-1054), CD44 (polyclonal rabbit anti-CD44, Abcam Cat No: Ab24504), CD29 (monoclonal rabbit anti-CD29, Millipore Cat No: 041109), CD146 (monoclonal rabbit anti-CD146, Millipore Cat No: 04-1147), Sca1 (rabbit polyclonal ant-Sca1, Millipore Cat No: AB4336), c-kit (polyclonal rabbit anti-c-Kit, Bioss Cat No: bs-10005R, polyclonal rabbit anti-c-Kit, Santa Cruz Cat No: sc-168) and flt-1 (monoclonal rabbit anti-Flt-1 Abcam Cat No: ab32152) and $\beta$-actin (monoclonal mouse anti- $\beta$-actin, Sigma Cat No: A5316).

\section{Cell culture}

A10 and A7r5 cells were obtained from ATCC Rockville, MD. Rat aortic SMC [rSMCs, R354-05a] were obtained from Cell Applications, CA. Cells were maintained in either Dulbecco's Modified Eagle's Medium (DMEM) or RPMI 1640 media supplemented with $10 \%$ foetal bovine serum (FBS), 150 units $/ \mathrm{ml}$ penicillin, and $150 \mu \mathrm{g} / \mathrm{ml}$ streptomycin (P/S) as previously described [ 21]. Cells were grown at $37^{\circ} \mathrm{C}$ in $5 \% \mathrm{CO}_{2}$ and $95 \%$ air. Confluent cells were passaged using $2 \mathrm{x}$ trypsin/0.53 mM EDTA. Gibco rat mesenchymal stem cells (MSCs) were obtained from Life Technologies, CA. MSC cells were maintained in growth media made up of 50:50 minimal essential medium ( $\alpha$-MEM) and Ham's F12 supplemented with $10 \%$ MSC defined FBS, $150 \mathrm{unit} / \mathrm{ml}$ penicillin, and $150 \mu \mathrm{g} / \mathrm{ml}$ streptomycin. Mesenchymal stem cells (MSCs) were characterised by differentiation along adipogenic lineages and the expression of cell surface markers indicative of MSC (i.e. CD29, CD44, CD90, CD146).

\section{Isolation of rat multipotent vascular stem cells [MVSCs]}

MVSCs were isolated from rat aortic explants as described previously [5]. Briefly, male Sprague Dawley rats were first anesthetized with pentobarbital sodium $(0.1 \mathrm{mg} / \mathrm{g})$ and then perfused with $10 \mathrm{~mL}$ of PBS. Arterial tissues were harvested as quickly as possible and further dissected in DMEM supplemented with $1 \%$ fetal bovine serum (FBS). The aorta was then isolated from the lower thoracic aorta to the upper abdominal aorta. The endothelium was removed by scraping off the cell 
layer on the luminal surface with sterile scalpel blade before the adventitia was carefully removed from media following brief enzymatic digestion with $2.5 \mathrm{mg} / \mathrm{mL}$ of collagenase for $15 \mathrm{~min}$ at $37^{\circ} \mathrm{C}$ using forceps under a dissection microscope. The remaining media was cut into $1-\mathrm{mm}$ pieces, placed onto the surface coated with $1 \%$ CellStart (Invitrogen) in 6-well plates and grown in MVSC culture medium containing DMEM with 2\% chick embryo extract (MP Biomedical), $1 \%$ FBS, 1\% N2 (Invitrogen), 2\% B27 (Invitrogen), $100 \mathrm{nM}$ retinoic acid (Sigma-Aldrich), $50 \mathrm{nM}$ 2-mercaptoethanol (SigmaAldrich), $1 \% \mathrm{P} / \mathrm{S}$ and $20 \mathrm{ngml}^{-1} \mathrm{bFGF}$ (R\&D Systems) (maintenance medium). All procedures were approved by the University Animal Care Committee and were carried out in accordance with EU guidelines for the Protection of Animals used for Scientific Purposes, (Amendment) Regulations 2013 (S.I. No 434 of 2013).

\section{Cell proliferation assays}

Cells were seeded at $1 \times 10^{4}$ cells/well and quiesced for $48 \mathrm{~h}$. The cells were then grown in DMEM supplemented with $10 \%$ FBS over 12 days. At each time point, the cells were washed twice in PBS and fixed in $4 \%$ paraformaldehyde (PFA) solution for 10 minutes at room temperature followed by 2 washes of PBS. Following the fixation process, cells were stained for 10 minutes in PBS containing $0.1 \%$ Triton X-100 with $30 \mu \mathrm{M}$ DAPI. Fluorescent images were collected by an Olympus DP-50 fluorescent microscope with the appropriate excitation and emission spectra at $4 \times, 10 \times, 20 \times$ magnification before the fluorescent DAPI labeled nuclei were the counted automatically using the Fiji software package [ 22].

\section{Immunoblot analysis}

Protein extracts $(15-40 \mu \mathrm{g})$ were fractionated by SDS-PAGE on 7-15\% (w/v) polyacrylamide resolving gels, as previously described [23]. Minor differences in protein loading and transfer were normalised using a Ponceau S stain and by measuring the constitutive $\beta$-actin protein levels.

\section{Quantitative real-time quantitative RT-PCR}

Quantitative real-time RT-PCR was performed using the Rotor Gene (RG-3000, Corvett Research) and the SYBR green PCR kit (Qiagen) according to the manufacturer's instructions. Cells were initially seeded onto 35mm culture dishes at a density of $1 \times 10^{5}$ cells/well in DMEM with $10 \%$ FBS, $1 \%$ penicillin/streptomycin and quiesced for 24 hours. Cells were then subjected to conditions of serum and serum deprivation in DMEM with 5\% FBS, $1 \%$ P/S and DMEM with $0.5 \%$ FBS, $1 \% \mathrm{P} / \mathrm{S}$ respectively for 48 hours. RNA was isolated using the Maxwell ${ }^{\mathrm{TM}} 16$ Total RNA Purification kit according to the manufacturer's instructions. RNA samples were quantified and investigated for purity using the nanodrop 2000 spectrophotometer (Thermo Scientific). Total RNA (1-10 ng) was then reverse transcribed and PCR amplified in a one-step reaction containing RT mix, SYBR green mix, and RNase free water at $55^{\circ} \mathrm{C}$ for $10 \mathrm{mins}, 95^{\circ} \mathrm{C}$ for 5 mins, followed by 60 cycles of $95^{\circ} \mathrm{C}$ for 5 seconds, $60^{\circ} \mathrm{C}$ for 15 seconds and subsequent melt curve an alysis. Samples were run in triplicate with a no reverse transcriptase (-RT) control. Gene expression was normalised to that of the housekeeping gene, GAPDH.

\section{Flow cytometry}

Cells were cultured until confluency in T75 flasks and then trypsinised in $2 x$ trypsin $/ 0.53 \mathrm{mM}$ EDTA at $37^{\circ} \mathrm{C}$. Cells were resuspended in media, counted, and then fixed in BD Cytofix/Cytoperm solution (BD Bioscience) for 20 mins at $4^{\circ} \mathrm{C}$. Cells were then washed in 1X BD Perm/Wash solution (BD Bioscience) by centrifugation at $500 \mathrm{~g}$ for $3 \mathrm{mins}$. Following washing, cells were resuspended in $1 \mathrm{X}$ BD Perm/Wash solution containing $1 \mu \mathrm{g}$ of appropriate primary antibody and incubated at $4^{\circ} \mathrm{C}$ for 30 mins. Following washing, cells were resuspended in 1X BD Perm/Wash solution containing $1 \mu \mathrm{g}$ of appropriate enzyme-linked secondary antibody and incubated at $4^{\circ} \mathrm{C}$ for 30 mins. Cells were then washed in BD Perm/Wash solution and resuspended in a final volume of $500 \mu \mathrm{L}$ of BD Perm/Wash solution.

\section{Immunocytochemistry}

Cells were seeded at a density of $1 \times 10^{4}$ cells/well onto non-coated glass cover slips $(20 \mathrm{~mm})$ (Thermo-Scientific) in 35 $\mathrm{mm}$ culture dishes and quiesced by serum deprivation for 24 hours. Cover slips were sterilised in IMS and washed twice in PBS prior to culturing. Media was then replaced to media containing either media containing $0.5 \%$ or $10 \%$ FCS and cells were cultured for a further 48 hours. Cells were washed twice in PBS and fixed for 15 min at room temperature (RT) with $3.7 \%$ paraformaldehyde prepared in PBS, washed twice with PBS, and then permeabilised by a 15 min RT exposure to a $0.1 \%$ Triton X-100/PBS solution. The cells were washed, blocked for 1 hour with a PBS solution containing 5\% BSA and $1 \%$ Tween before treatment with specific primary antibodies. Secondary antibodies were Alexa Fluor 546 goat anti-rabbit, rabbit anti-mouse and goat anti-mouse (Invitrogen) and Alexa Fluor 488 goat anti-rabbit (Invitrogen). Nuclei were stained with 4, 6-diamidino-2-phenylindole (DAPI) at a concentration of $2 \mu \mathrm{g} / \mathrm{ml}$ in PBS at RT for 10 minutes. Fluorescent images were collected by an Olympus DP-50 fluorescent microscope with the appropriate excitation and emission spectra at $4 \times, 10$ $\times, 20 \times$ and $60 \times$ magnification. Non-specific labeling was assessed following secondary antibody treatment.

\section{Confocal microscopy}

A7r5 and A10 cells were plated onto glass coverslips, placed in six well culture plates and returned to the incubator for a minimum of $24 \mathrm{~h}$ to allow for cell attachment and spreading. Cells were fixed and permeabilised by the addition of ice-cold acetone for $1 \mathrm{~min}$. The cells were then washed multiple times (3X) with phosphate-buffered saline (PBS) containing $0.5 \%$ TWEEN-20 (PBS-T); pH 7.5, and incubated for 10 min in blocking solution (5\% non-fat dry milk in PBS-T). Cells were stained for $30 \mathrm{~min}$ at room temperature with specific antibodies followed by incubation with an Alexa 488-labelled secondary antibody (Molecular Probes, Eugene, OR, USA). The cells on cover slips were mounted on slides with antifade medium (Dako). Slide preparations were observed using a Zeiss Axio Observer. Z1 equipped with a Zeiss 710 and 
ConfoCor3 laser scanning confocal head (Carl Zeiss, Inc.). Images were analyzed using Zen 2008 software as previously described [24].

\section{Adipocyte differentiation}

Cells were seeded onto 6-well-plates at a density of 50,000 cells/well. Cells were allowed to recover from trypsinisation for 2 days in complete medium. After recovery, cells were cultured in adipocyte differentiation media for $14 \mathrm{~d}$ (StemPro® Adipogenesis Differentiation, Life Technologies) as according to the manufacturer's instructions. Adipocyte differentiation was evaluated by Oil Red O and HCS LipidTOX ${ }^{\mathrm{TM}}$ Green neutral lipid staining (InVitrogen), as described by the manufacturers protocols.

\section{Osteoblast differentiation}

Cells were seeded onto 6-well-plates at a density of 50,000 cells/well and allowed to recover from trypsinisation for $2 \mathrm{~d}$ in maintenance medium. After recovery, cells were cultured in StemPro® Osteogenesis Differentiation Media Kit, Life Technologies) according to the manufacturer's instructions. Osteoblast differentiation was evaluated using 2\% Alizarin Red $\mathrm{S}$ stain (Sigma).

\section{Notch inhibition}

Cells were seeded at a density of $1.5 \times 10^{4}$ cells/well onto non-coated glass cover slips $(20 \mathrm{~mm})$ in 35 mm culture dishes and cultured for 72 hours in DMEM containing $10 \%$ FBS and $1 \%$ P/S. Cells were then treated with either DMSO (0.1\%) or the $\gamma$-secretase inhibitor, DAPT $(10 \mu \mathrm{M})$ for 48 hours. Cells were fixed for immunostaining as described above. DAPT, at a stock concentration of $18 \mathrm{mg} / \mathrm{ml}$, was diluted to a $10 \mu \mathrm{M}$ working solution in DMEM containing $10 \% \mathrm{FBS}$ and $1 \% \mathrm{P} / \mathrm{S}$.

\section{Statistics}

Results are expressed as mean $\pm \mathrm{SEM}$. Experimental points were performed in triplicate, with a minimum of three independent runs. A t-test was used for comparison of two groups. A value of $p \leq 0.05$ was considered significant.

\section{Results}

A10 and A7r5 cells were cultured in normal DMEM media supplemented with 10\% FCS before phenotypic analysis, based on SMC differentiation marker expression, was performed. The embryonic A10 cell line had the typical myoblast morphology when grown in culture and was positive for SMC differentiation markers SMA, CNN1 and SM-MHC (Figure 1 A). In addition, every cell appeared positive for each of these antigens. In contrast, embryonic A7r5 cell line had a flat ribbon-like structure and grew to parallel arrays of spindle shaped cells when confluent (Figure $1 \mathrm{~B}$ and C). Moreover, while these cells were also positive for SMC differentiation markers SMA, CNN1 and SM-MHC, there was a proportion of A7r5 cells that appeared to be weakly positive for SM-MHC and CNN1 when compared to the proportion of SMA positive cells (Figure 1 B). Figure 1

Vascular SMC Differentiation Marker expression. Aand B. Representative immunocytofluorescence of SMC differentiation markers in A10 and A7r5, respectively. Cells were cultured in DMEM supplemented medium for 3 days before immunocytochemistry was performed. Cells stained positive for (a-c)SMA (d-f)SM-MHC, and (g-i)CNN1. The nuclei of were stained with DAPI. Scale bar applies to all images, $250 \mathrm{nM}$. C.Confocal immunocytochemical staining of SMC differentiation markers in A7r5 cells. Quiesced A7r5 cells and grown in DMEM supplemented with (a-c, g-I, m-o)5\% FBS and (d-f, j-1, p-r) $0.5 \%$ FBS for $72 \mathrm{~h}$ before cells were analyzed under confocal immunofluorescence microscopy for SMC markers (a-f)SMA and (g-l)SM-MHC and (m-r)CNN1. Arrows designate myofilamentous structures. The nuclei of were stained with DAPI. Scale bar, $100 \mathrm{~nm}$ and $250 \mathrm{~nm}$. Data are representative of individual slides with similar results.

Quiescence of SMCs by serum deprivation has previously been shown to drive SMC differentiation with increased expression of SMA, CNN1 and SM-MHC reported [ 13]. We evaluated the repercussions of serum-deprivation on A10 and A7r5 SMC differentiation by measuring changes in the distribution and expression of SMC differentiation markers (SMA and CNN1) by confocal microscopy. In the case of A7r5 cells, there was a notable redistribution of SMA and CNN1 filaments in cells following serum-deprivation for $72 \mathrm{~h}$ when compared to serum conditions (Figure $1 \mathrm{C}$ ). Confocal immunocytofluorescence demonstrated a marked reorganization of actin filaments under serum conditions, compared with serum deprivation, with less filamentous staining and greater intensity at the periphery (Figure $1 \mathrm{C}$ ). In a similar manner, the distribution and filamentous nature of CNN1 staining was greater in serum-deprived cells when compared to serum conditions [Figure 1 C]. In contrast, there was no significant change in SM-MHC distribution (Figure 1 C). A similar profile with regard to the reorganization of SMA and CNN1 filaments and their filamentous nature was evident in A10 cells in response to serum deprivation (data not shown).

Previous studies had suggested that SM-MHC negative (SM-MHC ${ }^{-}$) cells can be defined as synthetic and/or proliferative SMCs in culture [ 4,14 ]. More recent studies now suggest that SM-MHC ${ }^{-}$cells derived from the vascular media by explant culture are multipotent vascular stem cells [MVSCs] capable of transition to several different non-vascular lineages [ 5, 15]. Therefore, MVSCs isolated from rat aortic explants as described previously [ 5], served as our control MVSC population. Microscopic analysis confirmed that the MVSCs were immunocytochemically negative for SM-MHC, but positive for neural stem cell markers Sox10, Sox17 and S100ß (Figure 2 A). The MVSCs also expressed several MSC-like phenotypic markers CD44 and CD29 in culture but were negative for CD146 [data not shown]. FACS analysis confirmed 
that the MVSCs were positive for Sox 10, Sox 17 and S100 $\beta$ (Figure 2B) but negative for the stage specific embryonic antigen (SSEA-1) marker. Figure 2

Multipotent vascular stem cell (MVSC) marker expression. A. Representative immunocytofluorescence of SMC differentiation markers (a-c)SM-MHC and neural stem cell markers (d-f)Sox10, (g-i)S100 $\beta$ and (j-1)Sox 17 in markers rat aortic MVSCs isolated by explant and cultured in MVSC maintenance media. The nuclei of were stained with DAPI. Scale bar, $100 \mathrm{~nm}$. Data are representative of three experiments. B. Representative flow cytometry analysis of MVSCs cultured in DMEM supplemented with 10\% FBS for $10 \mathrm{~d}$ with antibodies against SSEA-1, Sox 10, Sox 17 and S100 $\beta$. Open curves represent negative control samples; red filled curves represent samples stained with antibodies against SM-MHC, Sox 10, Sox 17 an $\mathrm{S} 100 \beta$.

While the MVSCs were immunocytochemically Sox $10^{+}$and SM-MHC ${ }^{-}$, they expres sed SMA in both maintenance and DMEM supplemented media [data not shown]. Moreover, the expression of SMC differentiation markers, SM-MHC and CNN1, was greatly enhanced following culture of these cells for $7 \mathrm{~d}$ in DMEM media supplemented with 10\% FBS (differentiation media) in the presence of the inductive stimuli, TGF- $\beta 1(2 \mathrm{ng} / \mathrm{ml})$ and PDGF $(10 \mathrm{ng} / \mathrm{ml})$ when compared to differentiation media over the same time period (Figure 3). FACS analysis of MVSCs further confirmed that these cells remain Sox $10^{+}$and Sox $17^{+}$positive while concomitantly expressing SM-MHC after $7 \mathrm{~d}$ [data not shown]. Figure 3

MVSC transition to vSMC following inductive stimulation.Representative immunocytofluorescence of SMC differentiation markers (a, b)SM-MHC, $(\mathrm{c}, \mathrm{d}) \mathrm{SMA}$ and $(\mathrm{e}, \mathrm{f}) \mathrm{CNN}{ }_{1}$ in MVSCs cultured in differentiation media (DMEM supplemented with $10 \%$ FBS) for $7 \mathrm{~d}$ in the absence or presence of $2 \mathrm{ng} / \mathrm{ml}$ TGF- $\beta 1$ and $10 \mathrm{ng} / \mathrm{ml}$ PDGF. The nuclei stained with DAPI. Scale bar, $100 \mathrm{~nm}$.

We therefore used anti-Sox10 and anti-SM-MHC antibodies to investigate the presence of 'MVSC-like' positive cells (SM$\mathrm{MHC}^{-}$Sox $10^{+}$) in each of the embryonic SMC cell lines. Using immunocytochemistry, we found no evidence for SM$\mathrm{MHC}^{-}$cells present in A10 cultures (Figure 4). In contrast, all of the cells stained strongly for SM-MHC ${ }^{+}$and uniformly expressed markers including neural crest cell markers Sox 10 and the endoderm marker Sox 17 (Figure 4A). These cells were also positive for stem cell antigen, $\mathrm{Sca}^{+}$. Subsequent FACS analysis of SMC differentiation and MVSC marker expression in these A10 cells confirmed quantitatively that $>90 \%$ of the cells were $\mathrm{MHC}^{+}$Sox $10^{+}$, Sox $17^{+}, \mathrm{S} 100 \beta^{+}$and $\mathrm{Sca}^{+}$(Figure 4B). Figure 4

MVSC marker expression in A10 cells. A. Representative immunocytofluorescence of neural stem cell markers (a-c)Sox10, (d-f)SM-MHC (g-i)Sox 17 and (j-1)Sca1 in quiesced cells grown in normal DMEM media supplemented with FBS for $3 \mathrm{~d}$ and visualised by (a-c)immunocytochemistry (d-i)confocal microscopy. Arrows show localisation of Sox10 and Sox17 primarily to the nucleus. The nuclei were stained with DAPI. Scale bar applies to all images, $250 \mathrm{~nm}$ except (a-c, $100 \mathrm{~nm})$. Data are representative of three individual slides. B. Representative flow cytometry analysis of A10 cells with antibodies against SM-MHC, Sox10, Sox 17, S100 $\beta$ and Sca1. Open curves represent negative control samples; red-filled curves represent samples stained with antibodies against SM-MHC, Sox10, Sox17, S100 $\beta$ and Sca1.

A7r5 cells were also positive SM-MHC, albeit not as robust as A10 cells, and were SM-MHC by FACS analysis. A7r5 were also positive for neural stem cell markers Sox $10^{+}$, Sox $17^{+}$and $\mathrm{S} 100 \beta^{+}$(Figure $5 \mathrm{~A}$ ). These cells were also positive for $\mathrm{Sca}^{+}$(Figure $5 \mathrm{~A}$ ). Subsequent FACS analysis confirmed quantitatively that $>90 \%$ of the cells were $\mathrm{MHC}^{+} \mathrm{Sox}_{10} 0^{+}$, Sox $17^{+}, \mathrm{S} 100 \beta^{+}$and Sca $1^{+}$(Figure 5 B). Parallel Western blot analysis of SMC differentiation and MVSC stem cell marker expression confirmed that both A10 and A7r5 cells express SMA, CNN1 and the MVSCs markers. However, the SM-MHC isoforms for A10 and A7r5 were different. The A10 cells expressed Sm2 whereas the A7r5 cells predominantly expressed non-muscle MHC, as previously reported [ 16, 17]. The MVSCs derived from rat aorta expressed Sm1 [Figure $6 \mathrm{~A}]$. In addition, the A10 and A7r5 cells both expressed c-kit and Flt-1 by immunocytochemistry and by FACS analysis (Figure 6B and C). However, unlike A10 and A7r5, MVSC and rSMCs were both positive for c-kit but weakly expressed flt-1 (Figure 6B and C). Figure 5

MVSC marker expression in A7r5 cells. A. Representative immunocytofluorescence of neural stem cell markers (ac)Sox10, (d-f)SM-MHC (g-i)Sox 17 and (j-1)Sca1 in quiesced cells grown in normal DMEM media supplemented with FBS for $3 \mathrm{~d}$ and visualised by (a-c)immunocytochemistry (d-i)confocal microscopy. Arrows show localisation of Sox 10 and Sox 17 primarily to the nucleus. The nuclei were stained with DAPI. Scale bar applies to all images, $250 \mathrm{~nm}$ except (a-c, $100 \mathrm{~nm}$ ). Data are representative of three individual slides. B. Representative flow cytometry analysis of A7r5 cells with antibodies against SM-MHC, Sox 10, Sox 17, S100 $\beta$ and Sca1. Open curves represent negative control samples; red-filled curves represent samples stained with antibodies against SM-MHC, Sox10, Sox 17, S100 $\beta$ and Sca1.

Figure 6

SMC and stem cell marker expression in A10, A7r5 and MVSCs. A. Representative immunoblots for SMC differentiation markers (SM-MHC, SMA and CNN1) and neural stem cell markers (Sox10, Sox 17, S100 $\beta$ in lysates from quiesced cells grown in normal DMEM media supplemented with FBS for $3 \mathrm{~d}$. The levels of SM-MHC (Sm1, Sm2 and non-muscle MHC), SMA, CNN1, Sox 10, Sox 17 and S100 $\beta$ were determined using specific antisera against these antigens. Data are representative of blots with similar results. Equal loading was confirmed by Ponceau S staining of the membranes and by measuring the constitutive $\beta$-actin gene. B. Immunocytochemical staining of c-Kit and Flt-1 in MVSCs, rSMC, A7r5 and A10 cells. Quiesced cells were grown in normal DMEM media supplemented with FBS for $3 \mathrm{~d}$ before the cells were stained for c-kit and flt-1. C. Representative flow cytometry analysis of Flt-1 and c-Kit in A7r5, A10 and MVSCs using antibodies against Flt-1 and c-Kit. Open curves represent negative control samples; red filled curves represent samples stained with antibodies.

To further investigate the expression of MVSC markers within the A10 and A7r5 cell lines, we carried out gene expression analysis of SMC differentiation and MVSC marker expression by real-time qRT-PCR. The repercussions of serum 
deprivation on SMC differentiation and MVSC marker expression in A7r5 cells were evaluated by measuring changes in SMC differentiation [SMA, Sm1, Sm2 and CNN1] and neural crest stem cell [Sox10, Sox17, S100 $\beta$ ] marker gene expression. Serum stimulation resulted in a significant increase in Sox10, Sox17 and S100 $\beta$ for A7r5 cells with a concomitant decrease in the SMC differentiation markers Sm1 and CNN1 while SMA and Sm2 levels increased. The increase in marker expression was greatest for S100 $\beta$ (Figure 7A). In contrast, serum stimulation increased A10 SMC differentiation marker expression with a concomitant increase in neural crest cell markers Sox 10, Sox 17 and S100 $\beta$ expression in these cells (Figure 7B). In addition, the growth of MVSCs lag behind the A10 and A7r5 in the early growth phase, but recover by day 12 [Figure $7 \mathrm{C}$ ]. Figure 7

Quantitative qRT-PCR of neural stem cell marker and SMC differentiation marker mRNA levels. Aand B. Quiesced A7r5 and A10 cells were cultured in DMEM media supplemented with 5\% FBS and 0.5\% FBS for $3 \mathrm{~d}$ before relative gene expression of SMC markers [SMA, MHC and CNN1] and neural stem cell MVSC markers [Sox10, Sox17 and S100 $\beta$ ] were determined by qRT-PCR. GAPDH was used to normalize gene expression. Data are mean \pm SEM and are representative of three independent wells, ${ }^{*} \mathrm{p}<0.01$ when compared to $0.5 \%$ FCS. C. Representative growth curves for A10, A7r5 and MVSCs grown for $12 \mathrm{~d}$ in their respective media. Cell number was quantified by counting DAPI stained nuclei.

Expression of neural stem cell markers [Sox10, Sox17 and S100 $\beta$ ] suggests that these embryonic SMC cell lines may retain some stem cell properties. To assess whether A10 and A7r5 cell lines were multipotent, we determined their capability of becoming adipocytes following treatment with specific induction media. Adipocyte differentiation was determined by both oil red staining and lipidTOX ${ }^{\mathrm{TM}}$ fluorescent staining after a $14 \mathrm{~d}$ treatment with the induction media. Rat mesenchymal stem cells (rMSCs) and rat aortic derived MVSCs (rMVSCs) were also treated with the same adipocyte induction media and served as positive controls (Figure 8). Bovine aortic endothelial cells served as a negative control (data not shown). Both rat MSCs and MVSCs were capable of differentiation to adipocytes after 14d treatment since the number of Oil Red O positive and LipidTOX ${ }^{\mathrm{TM}}$ positive cells was significantly increased (Figure 8). In parallel studies, rat MSCs and MVSCs were also capable of differentiation to osteoblasts after $14 \mathrm{~d}$ inductive treatment when analyzed with Alizarin Red S [data not shown]. While both A10 and A7r5 cells did retain some Oil Red O stain and LipidTOX ${ }^{\mathrm{TM}}$ fluorescent stain following treatment with inductive differentiation media, the number of positive cells was significantly less than MVSCs or the MSCs (Figure 8 ). Figure 8

Multipotent potential of MSC, MVSC, A10 and A7r5 cells in vitro .Representative images of adipocyte differentiation of MSCs, MVSCs and A10 and A7r5 cells following treatment of cells with adipocyte differentiation media for $14 \mathrm{~d}$. Adipocyte differentiation was determined by both Oil Red O and LipidTOX ${ }^{\mathrm{TM}}$ staining of lipid droplets. Data are representative of three independent experiments.

As Notch is a critical arbiter of mesenchymal stem cell (MSC) transition to vascular lineages [ 25, 26] and since MVSC transition to a MSC-like intermediate occurs en route to their differentiation to vSMC [ 5], we examined the effect of Notch inhibition on A10 and A7r5 differentiation. Notch 1 receptors, like in rat [ 27] and human vSMC [ 28], were present on these cells [data not shown]. We investigated if Notch inhibition following treatment with a $\gamma$-secretase inhibitor (DAPT), could force these cells to adopt a more contractile phenotype and decrease MVSC marker expression by monitoring SMMHC and Sox 10 expression using immunocytochemistry and qRT-PCR. We found that treatment of both cell types with DAPT did not significantly alter the percentage of Sox $10^{+}$, despite a change in cell number following Notch inhibition (Figure 9A, B). Moreover, Notch inhibition significantly enhanced MVSC marker expression [Sox10, Sox 17 and S100ß] in both cell types while preferentially increasing Sm2 and SMA in A7r5 but SMA, Sm1, Sm2, SMO and CNN1 in A10 cells (Figure 9A, B). Figure 9

The effects of Notch inhibition with DAPT on Sox 10 expression in A7r5 and A10 cells. A. Quiesced A10 cells were grown in media containing 10\% FBS supplemented with or without $10 \mu \mathrm{M}$ DAPT for $3 \mathrm{~d}$ before the expression of Sox 10 was determined by immunocytochemistry. B. Relative gene expression of SMC markers [(SMA, MHC, CNN1 and smoothelin (SMO)] and neural stem cell MVSC markers [Sox10, Sox17 and S100ß)] in A10 and A7r5 cells was determined by qRTPCR. GAPDH was used to normalize gene expression. Data are mean \pm SEM and are representative of three independent wells, $* \mathrm{p}<0.01$ when compared to $0.5 \%$ FCS.

\section{Discussion}

The characteristics of vSMCs and their modulation in culture have long been the subject of investigation [ 2,29]. The advent of stem cell derived SMCs has opened up a new debate about the phenotype and origin of 'modulated' dedifferentiated, neointimal cells in vivo and in vitro [ 15, 30-32]. Accordingly, we performed a comprehensive analysis of SMC differentiation and MVSC marker profile, growth properties and multipotency capacity for embryonic A7r5 and A10 SMC cells in culture.

The embryonic cell lines A7r5 and A10, and adult aortic SMC cells have all been shown to express SMC differentiation markers including SMA, CNN1, SM22, tropoelastin and to a lesser extent, SM-MHC [ 17, 33]. Interestingly, A10 and to some extent A7r5 resembled the epithelioid phenotype similar to that of the SMC population ("pup cells") cultured from the intimal thickening 15 days after endothelial injury [ 34]. A10 and A7r5 were also similar to cloned newborn rat SMC in that they continue to express SMA and SM-MHC in culture [33]. Both cell lines express abundant SMA and CNN1 with similar growth characteristics. Moreover, in agreement with previous studies of cultured SMC, the filamentous profile for SMA and CNN1 was enhanced under conditions of serum deprivation [ 12]. However, the expression of SM-MHC was noticeably different $[17,18]$. In the case of A10, the cells predominantly expressed Sm2 with some myofilamentous structures evident under both serum and serum-deprived conditions. In contrast, A7r5 cells predominantly expressed non- 
muscle SM-MHC with no discernible myofilaments. However, A7r5 have several characteristics in common with neointimal cells including the expression of SMA and non-muscle MHC [ 16].

The stem cell origin of vSMCs within vascular lesions has been controversial [ 30- 32]. Initial studies in canine carotid arteries suggested that neointimal modulated proliferative SMC were not derived from differentiated cells but instead formed by a myosin negative type 2 medial SMC cell [ 14]. Since then, several major types of resident SMC-related vascular progenitors have been identified within the tunica media. These include mesenchymal stem cell (MSC)-like cells, a side population of $\mathrm{Sca}^{+}$progenitors and multipotent vascular stem cells (MVSCs). MSC-like cells express CD29 and CD44 and have multilineage potential for osteogenic and chondrogenic differentiation but importantly, not for adipogenic differentiation [ 35]. MVSCs on the other hand express neural stem cell markers including Sox17, Sox10 and S100 $\beta$, are cloneable, have telomerase activity and can differentiate into neural cells and MSC-like cells that subsequently differentiate and transition to SMCs. Sca-1+ progenitors within the adventitia or media can differentiate into SMCs [29] and contribute to atherosclerosis of vein grafts in ApoE-deficient mice [ 20,36]. Further CD146 ${ }^{+}$perivascular MSC-like cells demonstrate osteogenic, chondrogenic and adipogenic potentials [37].

Importantly, lineage tracing has not resolved whether adult rat SMCs are derived from differentiated medial SMCs [ 9], MVSCs [5], or both. Elegant epigenetic signature studies tracking histone modifications of the Sm2 (MYH11) locus both in vitro and in vivo further suggest that SMCs both in culture and following injury are derived from mature differentiated SMC [ 11,38]. While the passage number, confluency and method of isolation for these SMCs i $n$ vitro may impact on these findings, it is clear that epigenetic signature studies are most useful when cells have lost a particular phenotype (and hence specific marker) or changed to multiple phenotypes. However, this is not the case in this study as A10 cells express both MVSC and SMC markers (Sm2 and non-muscle SM-MHC), respectively to varying degrees. Additionally, as MVSCs acquire SM-MHC (Sm2) expression when they are cultured in DMEM 10\% FBS media (and not maintenance media [ 11, $38]$ ), or induced using TGF- $\beta 1$, it is possible that the same histone modifications at the SM-MHC locus are apparent for MVSCs when acquiring SM-MHC expression.

The possibility also exists that de-differentiated SMC derived from differentiated medial SMC may revert back to multipotent/pluripotent stem cells and express neural stem cell markers. Indeed, recent studies have suggested that under certain circumstances, cultured rat SMC can be induced to osteogenic and skeletal muscle lineages [ 39, 40] further supporting a plasticity and stemness associated with SMC in culture that may involve acquiring neural stem cell markers. Indeed somatic cells in general can respond to stimulus-triggered acquisition of pluripotency [STAP] [ 41, 42]. Our study clearly demonstrates that both A10 and A7r5 uniformly express the neural stem cell markers [Sox10, Sox 17 and S100 3 ] typical of MVSCs [ 5] but also express Sca1, which is associated with perivascular adventitial progenitor stems cells [ 20]. They also concomitantly express SMC differentiation markers, SMA and CNN1 and Sm2 and non-muscle MHC, respectively [ 42]. Our data also suggest that both A10 and A7r5 cells also maintain some multipotent capabilities as they mimic MVSCs and MSC's in their ability to differentiate to adipocytes (and osteoblasts), albeit less robustly, following inductive stimulation. In addition, DAPT which inhibits Notch signalling and is a critical component during MSC to SMC transition [ 26, 43, 44] significantly alters both MVSC and SMC differentiation markers in both cell lines promoting neural stem cell phenotypes while increasing SMC differentiation. We have previously shown that Notch promotes SMC dedifferentiation in vitro [28]. It is also worth noting that mechanistically, Sox 17 acts upstream of the Notch system and downstream of the canonical Wnt system in orchestrating arterial specification during development and may thus be critical for MVSC transition to SMC in vitro [ 45].

The reason(s) for the appearance of MVSC neural stem cell markers in SMC cell lines in culture remain(s) unknown. One possibility is that both cell lines originate from MVSCs where the SMCs from MVSCs outgrow the de-differentiated SMC and eventually dominate the cultures [ 15]. In this regard, the growth curves for MVSCs, A10 and A7r5 suggest that MVSCs lag behind the embryonic cells in the early growth phase, but recover to comparable growth rates by day 12 . Notwithstanding the controversy as to whether neointimal SMC following injury are derived from resident stem cells or dedifferentiated SMCs, or even both, there is no controversy surrounding the phenotype of medial SMC in normal vessels in situ before culture. In this context, SMC prepared by explant culture from SM-MHC-Cre/LoxP-enhanced green fluorescence protein (EGFP) mice are predominantly immunocytochemically eGFP negative (and hence SM-MHC negative) but Sox 10, Sox 17 and S100 $\beta$ positive, yet acquire eGFP (SM-MHC) when sub-cultured in vitro or activated by a Notch ligand or TGF- $\beta 1$ in vitro [ 5] . In contrast, medial vSMC before enzymatic dispersal are predominantly eGFP (SMMHC) positive and express little to no Sox 10 , Sox 17 or S100 $\beta$ in situ. This profile is maintained when cells are enzymatically dispersed and cultured at P0 [ 15]. Over time, these cells are widely thought to become 'modulated' and reduce their expression of SMC differentiation markers, in particular SM-MHC, when compared to cells in situ or P0 in culture. Our data for rat aortic derived MVSCs suggests that cells maintained in stem cell maintenance media at early passage are predominantly SM-MHC $(\mathrm{Sm} 2)$ negative but positive for Sm1, Sox $10^{+}$, Sox $17^{+}$and S100 $\beta^{+}$. Importantly, they can transition to adipocytes (and osteoblasts) following specific in ductive stimulation and enhance their expression of SMC differentiation markers when grown in non-MVSC stem cell media (DMEM $+10 \%$ FCS or TGF- $\beta 1$ ).

The presence of stem cell antigen, $\mathrm{Sca} 1^{+}$on both embryonic SMC lines suggest that these cells also exhibit some perivascular markers. Vascular Sca $1^{+}$is associated with adventitial progenitors and a side population of medial progenitors that have the capacity of differentiate to SMC [ 6, 20, 29, 36]. While MVSCs are reported to be initially Sca1 ${ }^{-}$negative [ 5], the relationship, if any between MVSCs in the media and Sca ${ }^{+}$progenitors in the adventitia remains unknown. In addition, the presence of c-kit and Flt-1 positive cells within the A10, A7r5 and MVSC population is characteristic of SMCs derived from large arteries of older vessels and may suggest a similar stem cell origin [ 46].

In conclusion, the expression of neural stem cell markers in A10 and A7r5 suggests that these SMCs may represent MVSCderived SMC, de-differentiated SMC or both. In addition, their expression of perivascular Sca1 suggests that these cells may also have adventitial mesoderm origins. Both cell lines should now prove useful in determining the functionality of 
SMC in disease as they clearly resemble neo-intimal SMC known to be derived from MVSC following vascular injury [ 5]. Further single cell tracing experiments will be required to delineate the precise relationship between these two origins.

\section{Declarations}

\section{Acknowledgements}

This study was supported in part by funds from Science Foundation Ireland (SFI-11/PI/1128 to P.A. Cahill), the National Institutes of Health (R00HL095650 to D. Morrow and R21AA020365 to E.M. Redmond).

\section{Authors' original submitted files for images}

Below are the links to the authors' original submitted files for images.

Authors' original file for figure 1

Authors' original file for figure 2

Authors' original file for figure 3

Authors' original file for figure 4

Authors' original file for figure 5

Authors' original file for figure 6

Authors' original file for figure 7

Authors' original file for figure 8

Authors' original file for figure 9

Authors' original file for figure 10

Authors' original file for figure 11

Authors' original file for figure 12

Authors' original file for figure 13

Authors' original file for figure 14

Authors' original file for figure 15

Authors' original file for figure 16

Authors' original file for figure 17

\section{Competing interests}

On behalf of all authors, the corresponding author states that there is no competing interests.

\section{Authors' contributions}

EK, RH, CG and EM carried out the cell isolation, characterization and marker expression studies on each cell line. EK, CG, CM carried out the qRT-PCR analysis. EK, EM and SG performed the FACS analysis. EM, LEC and CEL carried out the confocal microscopic analysis. SG, DM EMR and PAC participated in the supervision of experiments and data analysis. EMR and PAC participated in the design, drafting and writing of manuscript. All auth ors have read and approved the final manuscript.

\section{References}

1. Chamley-Campbell J, Campbell GR, Ross R. The smooth muscle cell in culture. Physiol Rev. 1979;59:1-61. View Article Google Scholar

2. Orlandi A, Ehrlich HP, Ropraz P, Spagnoli LG, Gabbiani G. Rat aortic smooth muscle cells isolated from different layers and at different times after endothelial denudation show distinct biological features in vitro. Arterioscler Thromb. 1994;14:982-989.

View Article Google Scholar

3. Hao H, Gabbiani G, Bochaton-Piallat M-L. Arterial smooth muscle cell heterogeneity: implications for atherosclerosis and restenosis development. Arterioscler Thromb Vasc Biol. 2003;23:1510-1520.

View Article Google Scholar

4. Churchman AT, Siow RCM. Isolation, culture and characterisation of vascular smooth muscle cells. Methods Mol Biol. 2009;467:127-138.

View Article Google Scholar 
5. Tang Z Wang A Yuan F Yan Z Liu B Chu JS Helms JA Li S Differentiation of multipotent vascular stem cells contributes to vascular diseases Nat Commun 201238753538044 10.1038/ncomms1867

6. Torsney E, Xu Q. Resident vascular progenitor cells. J Mol Cell Cardiol. 2011;50:304-311. View Article Google Scholar

7. Xu Q. The role of stem cells in vein graft remodelling. Biochem Soc Trans. 2007;35:895-. View Article Google Scholar

8. Nguyen AT Gomez D Bell RD Campbell JH Clowes AW Gabbiani G Giachelli CM Parmacek MS Raines EW Rusch NJ Speer MY Sturek M Thyberg J Towler DA Weiser-Evans MC Yan C Miano JM Owens GK Smooth muscle cell plasticity: fact or fiction? Circ Res 201311217224135725 10.1161/CIRCRESAHA.112.281048

9. Nemenoff RA Horita H Ostriker AC Furgeson SB Simpson PA VanPutten V Crossno J Offermanns S Weiser-Evans MCM SDF-1 $\alpha$ induction in mature smooth muscle cells by inactivation of PTEN is a critical mediator of exacerbated injury-induced neointima formation Arterioscler Thromb Vasc Biol 2011311300 13083111081 10.1161/ATVBAHA.111.223701

10. Owens GK, Kumar MS, Wamhoff BR. Molecular regulation of vascular smooth muscle cell differentiation in development and disease. Physiol Rev. 2004;84:767-801.

View Article Google Scholar

11. Gomez D Owens GK Smooth muscle cell phenotypic switching in atherosclerosis Cardiovasc Res 201295 $156164338881610.1093 / \mathrm{cvr} / \mathrm{cvs} 115$

12. Li S, Sims S, Jiao Y, Chow LH, Pickering JG. Evidence from a novel human cell clone that adult vascular smooth muscle cells can convert reversibly between noncontractile and contractile phenotypes. Circ Res. 1999;85:338-348.

View Article Google Scholar

13. Su B, Mitra S, Gregg H, Flavahan S, Chotani MA, Clark KR, Goldschmidt-Clermont PJ, Flavahan NA. Redox regulation of vascular smooth muscle cell differentiation. Circ Res. 2001;89:39-46.

View Article Google Scholar

14. Holifield B Helgason T Jemelka S Taylor A Navran S Allen J Seidel C Differentiated vascular myocytes: are they involved in neointimal formation? J Clin Invest 199697814 825507120 10.1172/JCI118481

15. Tang Z, Wang A, Wang D, Li S. Smooth muscle cells: to be or not to be? Response to Nguyen et al. Circ Res. 2013;112:23-26.

View Article Google Scholar

16. Rao RS, Miano JM, Olson EN, Seidel CL. The A10 cell line: a model for neonatal, neointimal, or differentiated vascular smooth muscle cells?. Cardiovasc Res. 1997;36:118-126.

View Article Google Scholar

17. Firulli AB, Han D, Kelly-Roloff L, Koteliansky VE, Schwartz SM, Olson EN, Miano JM. A comparative molecular analysis of four rat smooth muscle cell lines. In Vitro Cell Dev Biol Anim. 1998;34:217-226. View Article Google Scholar

18. Graves DC, Yablonka-Reuveni Z. Vascular smooth muscle cells spontaneously adopt a skeletal muscle phenotype: a unique Myf5(-)/MyoD(+) myogenic program. J Histochem Cytochem. 2000;48:1173-1193. View Article Google Scholar

19. Nakajima S, Fujimoto M, Ueda M. Spatial changes of [Ca2+]i and contraction caused by phorbol esters in vascular smooth muscle cells. Am J Physiol. 1993;265:C1138-C1145.

View Article Google Scholar

20. Hu Y Zhang Z Torsney E Afzal AR Davison F Metzler B Xu Q Abundant progenitor cells in the adventitia contribute to atherosclerosis of vein grafts in ApoE-deficient mice J Clin Invest 20041131258 $126539842610.1172 / \mathrm{JCI} 19628$

21. Cappadona C, Redmond EM, Theodorakis NG, McKillop IH, Hendrickson R, Chhabra A, Sitzmann JV, Cahill PA. Phenotype dictates the growth response of vascular smooth muscle cells to pulse pressure in vitro. Exp Cell 
22. Schindelin J, Arganda-Carreras I, Frise E, Kaynig V, Longair M, Pietzsch T, Preibisch S, Rueden C, Saalfeld S, Schmid B, Tinevez J-Y, White DJ, Hartenstein V, Eliceiri K, Tomancak P, Cardona A. Fiji: an open-source platform for biological-image analysis. Nat Meth. 2012;9:676-682.

View Article Google Scholar

23. Gao W, Ferguson G, Connell P, Walshe T, Murphy R, Birney YA, O’Brien C, Cahill PA. High glucose concentrations alter hypoxia-induced control of vascular smooth muscle cell growth via a HIF-1alphadependent pathway. J Mol Cell Cardiol. 2007;42:609-619.

View Article Google Scholar

24. McEntee G Minguzzi S O’Brien K Ben Larbi N Loscher C O’Fágáin C Parle-McDermott A The former annotated human pseudogene dihydrofolate reductase-like 1 (DHFRL1) is expressed and functional Proc Natl Acad Sci USA 201110815157151623174580 10.1073/pnas.1103605108

25. Kurpinski K, Lam H, Chu J, Wang A, Kim A, Tsay E, Agrawal S, Schaffer DV, Li S. Transforming growth factorbeta and notch signaling mediate stem cell differentiation into smooth muscle cells. Stem Cells. 2010;28:734742.

View Article Google Scholar

26. Doi H, Iso T, Shiba Y, Sato H, Yamazaki M, Oyama Y, Akiyama H, Tanaka T, Tomita T, Arai M, Takahashi M, Ikeda U, Kurabayashi M. Notch signaling regulates the differentiation of bone marrow-derived cells into smooth muscle-like cells during arterial lesion formation. Biochem Biophys Res Commun. 2009;381:654-659. View Article Google Scholar

27. Sweeney C, Morrow D, Birney YA, Coyle S, Hennessy C, Scheller A, Cummins PM, Walls D, Redmond EM, Cahill PA. Notch 1 and 3 receptor signaling modulates vascular smooth muscle cell growth, apoptosis, and migration via a CBF-1/RBP-Jk dependent pathway. FASEB J. 2004;18:1421-1423.

View Article Google Scholar

28. Morrow D, Scheller A, Birney YA, Sweeney C, Guha S, Cummins PM, Murphy R, Walls D, Redmond EM, Cahill PA. Notch-mediated CBF-1/RBP-J\{kappa\}-dependent regulation of human vascular smooth muscle cell phenotype in vitro. Am J Physiol Cell Physiol. 2005;289:C1188-96.

View Article Google Scholar

29. Sainz J, Haj Zen Al A, Caligiuri G, Demerens C, Urbain D, Lemitre M, Lafont A. Isolation of "side population" progenitor cells from healthy arteries of adult mice. Arterioscler Thromb Vasc Biol. 2006;26:281-286.

View Article Google Scholar

30. Yu H, Stoneman V, Clarke M, Figg N, Xin H-B, Kotlikoff M, Littlewood T, Bennett M. Bone marrow-derived smooth muscle-like cells are infrequent in advanced primary atherosclerotic plaques but promote atherosclerosis. Arterioscler Thromb Vasc Biol. 2011;31:1291-1299.

View Article Google Scholar

31. Albiero M, Menegazzo L, Fadini GP. Circulating smooth muscle progenitors and atherosclerosis. Trends Cardiovasc Med. 2010;20:133-140.

View Article Google Scholar

32. Orlandi A, Bennett M. Progenitor cell-derived smooth muscle cells in vascular disease. Biochem Pharmacol. 2010;79:1706-1713.

View Article Google Scholar

33. Bochaton-Piallat ML, Gabbiani F, Ropraz P, Gabbiani G. Cultured aortic smooth muscle cells from newborn and adult rats show distinct cytoskeletal features. Differentiation. 1992;49:175-185.

View Article Google Scholar

34. Bochaton-Piallat ML, Ropraz P, Gabbiani F, Gabbiani G. Phenotypic heterogeneity of rat arterial smooth muscle cell clones. Implications for the development of experimental intimal thickening. Arterioscler Thromb Vasc Biol. 1996;16:815-820.

View Article Google Scholar

35. Tintut Y, Alfonso Z, Saini T, Radcliff K, Watson K, Boström K, Demer LL. Multilineage potential of cells from the artery wall. Circulation. 2003;108:2505-2510. 
36. Xu Q. Stem cells and transplant arteriosclerosis. Circ Res. 2008;102:1011-1024.

View Article Google Scholar

37. Crisan M, Yap S, Casteilla L, Chen C-W, Corselli M, Park TS, Andriolo G, Sun B, Zheng B, Zhang L, Norotte C, Teng P-N, Traas J, Schugar R, Deasy BM, Badylak S, Buhring H-J, Giacobino J-P, Lazzari L, Huard J, Péault B. A perivascular origin for mesenchymal stem cells in multiple human organs. Cell Stem Cell. 2008;3:301-313. View Article Google Scholar

38. Gomez D Shankman LS Nguyen AT Owens GK Detection of histone modifications at specific gene loci in single cells in histological sections Nat Methods 201310171 1773560316 10.1038/nmeth.2332

39. Ciceri P, Volpi E, Brenna I, Arnaboldi L, Neri L, Brancaccio D, Cozzolino M. Combined effects of ascorbic acid and phosphate on rat VSMC osteoblastic differentiation. Nephrol Dial Transplant. 2012;27:122-127.

View Article Google Scholar

40. Liao X-B, Zhang Z-Y, Yuan K, Liu Y, Feng X, Cui R-R, Hu Y-R, Yuan Z-S, Gu L, Li S-J, Mao D-A, Lu Q, Zhou XM, de Jesus Perez VA, Yuan L-Q. MiR-133a modulates osteogenic differentiation of vascular smooth muscle cells. Endocrinology. 2013;154:3344-3352.

View Article Google Scholar

41. Obokata H, Sasai Y, Niwa H, Kadota M, Andrabi M, Takata N, Tokoro M, Terashita Y, Yonemura S, Vacanti CA, Wakayama T. Bidirectional developmental potential in reprogrammed cells with acquired pluripotency. Nature. 2014;505:676-680.

View Article Google Scholar

42. Obokata H, Wakayama T, Sasai Y, Kojima K, Vacanti MP, Niwa H, Yamato M, Vacanti CA. Stimulus-triggered fate conversion of somatic cells into pluripotency. Nature. 2014;505:641-647.

View Article Google Scholar

43. Yamamizu K Matsunaga T Uosaki H Fukushima H Katayama S Hiraoka-Kanie M Mitani K Yamashita JK Convergence of Notch and beta-catenin signaling induces arterial fate in vascular progenitors J Cell Biol 20101893253382856895 10.1083/jcb.200904114

44. Kane NM, Xiao Q, Baker AH, Luo Z, Xu Q, Emanueli C. Pluripotent stem cell differentiation into vascular cells: a novel technology with promises for vascular re(generation). Pharmacol Ther. 2011;129:29-49. View Article Google Scholar

45. Corada M Orsenigo F Morini MF Pitulescu ME Bhat G Nyqvist D Breviario F Conti V Briot A IruelaArispe ML Adams RH Dejana E Sox 17 is indispensable for acquisition and maintenance of arterial identity Nat Commun 2013426093826640 10.1038/ncomms3609

46. Ferlosio A, Arcuri G, Doldo E, Scioli MG, De Falco S, Spagnoli LG, Orlandi A. Age-related increase of stem marker expression influences vascular smooth muscle cell properties. Atherosclerosis. 2012;224:51-57. $\underline{\text { View Article Google Scholar }}$ 